\title{
EDITORIAL
}

\section{Editorial PIAM January 2021}

\author{
Eujin Pei ${ }^{1}$
}

Accepted: 25 January 2021 / Published online: 6 February 2021

(c) The Author(s), under exclusive licence to Springer Nature Switzerland AG part of Springer Nature 2021

We start a brand-new year in 2021, with an exciting new author dashboard for submissions to Progress in Additive Manufacturing. At Springer Nature, we are committed to increase the level of transparency in our peer review process. We recognize that this process is important for authors and we have partnered with Research Square to provide a private dashboard system. We hope that corresponding authors will be able to receive a better insight into this process. Starting from January, corresponding authors will have real-time access to the status of their manuscript through a peer review timeline that displays the activities taking place, such as their manuscript being assigned to a reviewer or the receipt of a reviewer report. This private dashboard system will provide access to free, useful resources and other optional services to improve the manuscript and promote the publication. This includes an online manuscript editing service and research promotion services for video and visual abstracting.

For this issue, we have lined up a series of papers based on Material Extrusion that has been the mainstay of Additive Manufacturing. The articles include a paper on soybean hull fiber composite filaments, process parameters on mechanical properties for carbon fiber-PLA materials, the influence of stiffener geometry on flexural properties of PLA materials, mathematical design and mechanical analysis of lattice structures, the impact strength of PLA materials, the metalMaterial Extrusion process, and the modelling and optimization of Material Extrusion itself. This issue also includes a paper that proposes a software that seeks to predict the printability of soft materials, the tailoring of properties of photopolymers, and multi-objective optimization of machining factors. Lastly, we also present two review articles about the modelling of Material Extrusion; and a review of Additive Manufacturing during the COVID-19 pandemic.

I hope you will enjoy reading this issue. Write to us-we will be happy to hear from you.

Eujin Pei, Progress in Additive Manufacturing Journal, January 2021.

Publisher's Note Springer Nature remains neutral with regard to jurisdictional claims in published maps and institutional affiliations.

Eujin Pei

eujin.pei@brunel.ac.uk

1 Brunel University London, London, UK 